\title{
Convergência de renda e seus determinantes: uma ênfase na qualidade e na quantidade do capital humano dos municípios brasileiros
}

\author{
Joana Darc K. P. Honesko ${ }^{1}$ \\ Augusta P. Raiher ${ }^{2}$
}

\begin{abstract}
Resumo: Este artigo tem como objetivo analisar o efeito do capital humano - tanto quantitativo como qualitativo - no processo de crescimento da renda per capita dos municípios brasileiros nos anos 2000, testando a hipótese de convergência de renda. Para isso, além de se construir uma proxy quantitativa para o capital humano (escolaridade média do trabalhador), ponderou-a pela qualidade, média do IDH (Índice de Desenvolvimento Humano) longevidade com o IDH conhecimento. Na sequência, por meio da econometria espacial, testou-se os impactos da qualidade e da quantidade de capital humano no crescimento da renda per capita dos municípios brasileiros, reestimando para grupos de municípios (Norte/Nordeste versus Sul/Sudeste/Centro-Oeste). Como corolário, aqueles municípios que detinham uma renda menor no período inicial tenderam a ter um ritmo de crescimento subsequente maior, relação que se apresentou mais intensa quando se usou a qualidade como medida do capital humano. Ao mesmo tempo, a dinâmica do crescimento econômico tendeu a ser mais intensa quanto maior o estoque qualitativo e quantitativo do capital humano.
\end{abstract}

Palavras-Chave: Capital humano. Convergência de Renda. Econometria Espacial.

Income convergence and its determinants: an emphasis on the quality and quantity of the human capital of brazilian municipalities

\footnotetext{
Abstract: This article aims to analyze the effect of human capital - both quantitative and qualitative - in the process of per capita income growth of Brazilian municipalities in the year 2000, testing the hypothesis of income convergence. For

${ }^{1}$ Mestranda do Programa de Pós-Graduação em Economia da Universidade Estadual de Ponta Grossa (UEPG). Email: joana@balsano.com.br

${ }^{2}$ Professora do Programa de Pós-Graduação em Economia, do Programa de Pós-Graduação em Ciências Sociais e do departamento de economia da UEPG. Bolsista Produtividade CNPQ. E-mail: apelinski@gmail.com
} 
this, in addition to constructing a quantitative proxy for human capital (average worker education), weighted it by the quality (average of the Human Development Index - IDH - longevity with the IDH-knowledge). Afterwards, through spatial econometrics, it tested the impacts of the quality and quantity of human capital on the per capita income growth of Brazilian municipalities, reestimating to groups of municipalities (North / Northeast versus South / Southeast / Center-West). As a corollary, those municipalities that had a lower income in the initial period tended to have a higher growth rate in the following period, a relationship that was more intense when the measure of human capital was quality. At the same time, the dynamics of economic growth tended to be more intense the greater the qualitative and quantitative stock of human capital.

Keywords: Human capital; Income convergence; Spatial econometrics.

Classificação JEL: Rı.

1. Introdução

Todo elemento formador do conhecimento, das aptidões, que resulte num aumento da produtividade, das habilidades dos indivíduos, é entendido como capital humano (OCDE, 2001). Por isso, sua construção é cumulativa ao longo do tempo. Nesse contexto, o treinamento, a escolaridade, a cultura, a saúde, a alimentação, o ambiente familiar apresentam-se como fatores importantes desse processo (SCHULTZ, 1963).

Teoricamente, esses elementos melhoram as habilidades individuais acarretando numa intensificação da produtividade regional, decorrentes, basicamente, de três vias: por meio do aumento da produtividade do trabalhador; via o processo de inovação, e; por meio da difusão tecnológica (NAKABASHI e FIGUEIREDO, 2005).

Nelson e Phelps (1966) destacam que mesmo em funções rotineiras, trabalhadores com maior nível de capital humano geram uma produção mais elevada. Portanto, a capacitação dos indivíduos, com a formação do seu capital humano, torna-se crucial no desenvolvimento da sua produtividade. Ademais, dentro do processo de geração de conhecimento (inovação e difusão de tecnologia), organizações com maior capital humano detém potencial superior para produzir novos produtos e processos (CRAWFORD,1994). Assim, o capital humano torna-se essencial para transformar ideias e inovações em novos meios de 
produção e novos produtos, bem como para adaptar tais descobertas ao sistema produtivo vigente nas regiões.

Recentemente, alguns trabalhos apresentam-se como decisivos para o processo de crescimento econômico, não apenas a quantidade de capital humano que se tem, mas principalmente a sua qualidade. Hanushek e Kimko (2000), por exemplo, ao construir indicadores de qualidade do capital humano baseado em testes de proficiência internacional, argumentam que o papel das externalidades do capital humano para o crescimento econômico é significativamente mais forte para a qualidade da força de trabalho do que para a quantidade de capital humano. Ademais, seus resultados demonstram que a qualidade é significante para explicar disparidades de renda internacional.

Nakabashi e Figueiredo (2005) ponderam a escolaridade das Nações Unidas pelo Índice de Desenvolvimento Humano (IDH), incluindo aspectos qualitativos no capital humano. Como corolário, a qualidade do capital humano apresenta-se importante na dinâmica econômica, elevando também a confiabilidade das estimativas auferidas.

Barro (2000), utilizando uma amostra de cem países, direciona sua análise na relação entre educação e capital humano, distinguindo o efeito quantitativo (média de anos de estudo para diversos níveis) e o qualitativo (obtido por exames internacionais). Seus resultados evidenciam que o efeito da qualidade do ensino é quantitativamente mais relevante para o processo de crescimento econômico.

Connolly (2004), analisando 48 estados americanos, relaciona também a qualidade do capital humano com os fatores ligados à renda. Dentre os elementos relacionados à qualidade, elencam: o maior investimento no setor, os maiores salários dos professores, a carga horária escolar mais intensa e, o número menor de alunos por professores. O melhoramento desses elementos resultou numa relação positiva com a produtividade e com a experiência do trabalhador americano.

A nível nacional, Nakabashi e Salvato (2007) consideram o efeito individual da qualidade e da quantidade do capital humano na formação da renda por trabalhador dos estados brasileiros, utilizando as variáveis: número de alunos por sala de aula, percentual de professores com ensino superior completo e taxa de aprovação dos alunos. Verificam que um ano a mais de estudo sem mudanças na qualidade resulta num impacto menor na renda do que quando se considera simultaneamente a qualidade e a quantidade. Portanto, a qualidade do capital humano é uma característica importante na determinação dos efeitos desse fator sobre o nível e a taxa de crescimento da renda por trabalhador.

Raiher e Dathein (2009), analisando as microrregiões paranaenses, demonstram que a quantidade de capital humano (escolaridade média) não exerce efeito no dinamismo econômico do Paraná, ao mesmo tempo que as alterações na sua qualidade se apresentam decisivas para o processo. Para isso, construíram um 
índice da qualidade do capital humano, mensurado pelo percentual de professores da rede pública com ensino superior completo, pelo número médio de estudantes por sala de aula e pela nota média no Exame Nacional de Ensino Médio (Enem).

Igualmente, Firme e Simão Filho (2014) também buscam identificar o impacto da qualidade do capital humano na taxa de crescimento econômico dos municípios de Minas Gerais, utilizando duas proxies: escolaridade ponderada pelo IDH conhecimento e IDH saúde. Como resultado, identificam um efeito positivo e estatisticamente significativo para as duas proxies do capital humano. Ao trabalhar com regimes espaciais considerando as mesorregiões mineiras, corroborou tais inferências para a maior parte dos espaços.

No trabalho de Saraiva (2016), o autor mensura um índice de capital humano quantitativo (escolaridade média) e qualitativo (nota no Enem, nota da redação do Enem, mortalidade infantil, e infraestrutura escolar) para as microrregiões brasileiras, analisando a sua distribuição espacial, evidenciando uma heterogeneidade nessa distribuição.

Destarte, a não inclusão dos aspectos qualitativos do capital humano pode resultar em ausência de impacto dessa variável na dinâmica econômica. Islam (1995) e Woessmann (2003) relacionam a não significância do componente "capital humano" em muitos modelos de crescimento econômico como resultado das próprias proxies que são utilizadas, as quais não captam a qualidade do ensino, da formação profissional. Bosworth e Collins (2003) argumentam que os aspectos apenas quantitativos não incorporam elementos como a infraestrutura educacional, as condições de saúde, de bem-estar, parâmetros importantes na determinação do nível de produtividade, de conhecimento regional. Ou seja, ao ignorar os aspectos qualitativos, assume-se um mesmo impacto do aumento da escolaridade na produtividade regional, independente das condições locais de cada espaço.

Portanto, na investigação dos determinantes do crescimento econômico regional é importante incluir proxies representativa da qualidade do capital humano. E é nesse contexto que este trabalho se insere, buscando identificar o efeito que o capital humano - tanto quantitativo como qualitativo - exercem no processo de crescimento da renda per capita dos municípios brasileiros dos anos 2000, testando a hipótese de convergência de renda.

Ressalta-se que a maioria das evidências empíricas para o Brasil focam especialmente na quantidade de capital humano existente. Ademais, os poucos trabalhos que analisam o efeito da sua qualidade no crescimento econômico abrangem apenas os estados ou microrregiões brasileiras, ou municípios de apenas alguns estados.

Portanto, existe uma lacuna na literatura empírica nacional e esta pesquisa avança nesse sentido, abordando a problemática do crescimento econômico versus 
a qualidade do capital humano no âmbito dos municípios brasileiros nos anos 2000. A expectativa é de se ter resultados mais robustos, investigando, a priori, a desigualdade na distribuição da qualidade/quantidade do capital humano ao longo do país e, na sequência, a importância que esse fator produtivo detém na dinâmica econômica municipal. Mais do que isso, utilizar-se-á a análise espacial e com ela poder-se-á identificar os efeitos de externalidade do capital humano (em termos quantitativo e qualitativo) no crescimento da renda dos municípios do envoltório.

Assim, dois pontos diferenciam esta pesquisa: o nível de desagregação, abrangendo todos os municípios brasileiros, e a metodologia, utilizando a econometria espacial. Como o Brasil apresenta uma heterogeneidade na distribuição dos seus recursos produtivos, fez-se estimativas adicionais, usando uma variável categórica (discreta) para identificar regimes espaciais Sul/Sudeste/Centro-Oeste versus Norte/Nordeste. As evidências empíricas apresentadas ao longo do texto irão mostrar as diferentes dinâmicas de crescimento entre essas regiões (heterogeneidade espacial), justificando tal procedimento.

Para isso, este trabalho está dividido em quatro seções, incluindo esta. Na segunda, tem-se os elementos metodológico. A análise da distribuição espacial do capital humano e do crescimento da renda per capita, com a identificação do impacto da qualidade/quantidade de capital humano na dinâmica econômica e no processo de convergência é apresentado na terceira seção. Por fim, tem-se a conclusão.

\section{Elementos metodológicos: modelo e estratégia empírica}

Na década de 1980, importantes trabalhos discutiram os determinantes da distribuição da renda per capita entre os países. Dentre eles, tem-se o de Baumol (1986), no qual analisou o crescimento econômico e a convergência de renda de grupos de países. No caso dos desenvolvidos, as evidências estatísticas apontaram para uma associação inversa entre os níveis de produtividade em 1870 e suas taxas médias de crescimento da produtividade entre 1870 e 1979. Observou-se o mesmo resultado para os países com renda média. $\mathrm{O}$ único grupo no qual se teve divergência nos resultados foi para os países mais pobres. Com isso, Baumol (1986) inferiu que a convergência da produtividade ocorre especialmente nas economias industrializadas, convergência aparentemente compartilhada pelas economias planejadas.

Outro trabalho que investigou as diferenças de renda entre os países foi o de Mankiw, Romer e Weil (1992). Os autores testaram empiricamente o modelo de Solow e incluíram a acumulação de capital humano. Os resultados indicaram que o modelo de Solow não prediz convergência absoluta (convergência das rendas per 
capita de todos os países para o mesmo nível), mas sim convergência condicional, referindo-se à convergência para um mesmo nível de renda per capita dos países com taxas idênticas de investimento em capital físico e humano e idênticas taxas de crescimento da população.

A essência desse modelo enfatiza que a disparidade de renda entre regiões provém do nível inicial da renda de determinada localidade e da velocidade com que essa renda cresce em direção ao seu equilíbrio de longo prazo. Assim, tem-se a necessidade de entender a dinâmica da convergência da renda de equilíbrio de longo prazo para entender a diferença de renda entre as regiões.

Esse é o modelo testado para os municípios brasileiros, investigando se há convergência de renda, e se a qualidade/quantidade de capital humano impacta nesse processo. Por isso, nas subseções seguintes apresentar-se-á o modelo Mankiw, Romer e Weil (MRW), seguida da estratégica empírica utilizada para estimá-lo.

\subsection{Modelo MRW}

O modelo de Solow considera que o produto de uma economia é função do capital de trabalho. Nesse sentido, Solow (1956), partindo de uma função de produção do tipo Cobb-Douglas com retornos constantes de escala, considera que:

$$
Y=A K^{\alpha} L^{1-\alpha}
$$

Em que $K$ e $L$ são os níveis do fator capital e trabalho respectivamente, $A$ é um nível da tecnologia e $\alpha$ é um parâmetro que varia entre zero e um.

MRW (1990), utilizando dados pós-segunda guerra mundial de alguns países, testaram o modelo de Solow e observaram que o produto cresce com o aumento do capital físico e reduz quando se tem um crescimento populacional, mas apenas para alguns países: os de economia intermediária e os não produtores de petróleo.

Os autores incluíram então em (1) o capital humano, considerando que a exclusão deste poderia estar superestimando os coeficientes.

$$
Y=A K^{\alpha} H^{\beta} L^{1-\alpha-\beta}
$$

Em que $H$ representa o capital humano; $a$ e $b$ e eferem-se às elasticidades do produto em relação ao $\mathrm{Ke} \mathrm{L}$, respectivamente.

Expressando o produto e o estoque de capital físico e humano em unidades efetivas de trabalho e considerando que o capital físico e humano deprecia-se a uma mesma taxa, $d$, os autores deduzem a taxa de crescimento do capital físico e humano por trabalhador como:

$$
\dot{k}=s_{k} y-(n+g+\delta) k
$$




$$
\dot{h}=s_{h} y-(n+g+\delta) h
$$

Em que $s_{k}$ representa a fração da renda investida em capital físico, $s_{h}$ é a fração da renda investida em capital humano, $k$ refere-se ao capital por trabalhador, $h$ é o produto por trabalhador, $n$ é a taxa de crescimento populacional, $g$ é a taxa de crescimento da tecnologia, $\delta$ é a taxa de crescimento da depreciação. Ressalta-se que, segundo as suposições do modelo de Solow, $A$ e $L$ crescem a uma taxa $g+n$.

Após algumas manipulações algébricas e considerando que os países se encontram no estado estacionário, MRK (1990) chegam a seguinte equação:

$$
\ln y=\ln A+\left(\frac{\alpha}{1-\alpha-\beta}\right) \ln s_{k}+\left(\frac{\beta}{1-\alpha-\beta}\right) \ln s_{h}-\left(\frac{\alpha+\beta}{1-\alpha-\beta}\right) \ln (n+g+\delta)+\varepsilon
$$

Ao testar empiricamente (5), os autores identificaram que cerca de $80 \%$ da variação da renda per capita é explicado por essa equação, ressaltando que MRW (1990) encontraram evidências de forte influência do capital humano na diferenciação do crescimento entre os países. Observaram ainda que a renda no estado estacionário é diretamente correlacionada ao capital físico e humano e inversamente à taxa de crescimento populacional. A ausência do capital humano possivelmente geraria estimadores tendenciosos, tendo em vista que essa variável está correlacionada ao crescimento populacional e a taxa de poupança.

Em relação à convergência, como não se tem garantia de que os países estejam no estado estacionário, os autores relaxaram tal hipótese. Assim, considerando o período de convergência, obtém-se:

$$
\ln y_{t}=\left(1-e^{-1 t}\right) \ln y^{*}+e-{ }^{1 t} \ln y_{o}
$$

Sendo $y^{*}$ a renda do estado estacionário (em unidades efetivas de trabalho) e $\lambda$ a taxa de convergência, $\operatorname{com} \lambda=(n+g+\delta)(1-\alpha-\beta)$. Substituindo $y^{*} e$ subtraindo $y_{\mathrm{o}}$ de ambos os lados, matematicamente obtém-se:

$$
\ln \left(\frac{y_{t}}{y_{0}}\right)=\left(1-e^{-\lambda t}\right)\left[-\ln \left(y_{0}\right)+\frac{\alpha}{1-\alpha-\beta} \ln \left(s_{k}\right)-\frac{\alpha}{1-\alpha-\beta} \ln (n+g+\delta)+\frac{\beta}{1-\alpha-\beta} \ln \left(s_{b}\right)+\ln A_{0}\right.
$$

A suposição de (7) é de que as diferença na taxa de retorno e no movimento do capital - físico e humano - dos países pobres tenderiam a ser maiores. $\mathrm{O}$ modelo (7) pode ser apresentado como:

$$
\ln \left(\frac{y_{t}}{y_{0}}\right)=\beta_{0}+\beta_{1} \ln \left(y_{0}\right)+\beta_{2} \ln \left(s_{k}\right)+\beta_{3} \ln (n+g+\delta)+\beta_{4} \ln \left(s_{k}\right)+\varepsilon
$$


Em que $y_{t}$ é a renda per capita real; $y_{o}$ a renda per capital inicial; $s_{k}$ a fração da renda investida em capital físico e; $s_{h}$ a fração da renda investida em capital humano.

\subsection{Estratégia empírica: Econometria espacial}

O impacto do capital humano na convergência de renda dos municípios brasileiros foi estimado pelo modelo MRW (8). No entanto, é possível que a renda per capita do município $i$ afete positivamente o crescimento econômico dos seus vizinhos, da mesma forma que a formação do capital físico, do capital humano de um município, pode afetar o ritmo de crescimento do seu envoltório, resultado direto dos encadeamentos produtivos, dos spillovers tecnológicos, etc. Por isso, é necessário controlar esses fatores espaciais para se ter, de fato, os determinantes do crescimento econômico e da convergência de renda dos municípios brasileiros.

Por isso, a equação (8) foi estimada por meio da econometria espacial. Nesta metodologia, o primeiro passo é a decisão do melhor modelo espacial - Modelo de defasagem espacial (SAR), Modelo de erro autorregressivo espacial (SEM), Durbin espacial (SDM) ou Durbin espacial do erro (SDEM). Para isso, baseou-se na qualidade de ajuste do modelo, conforme os critérios de informação de Akaike e de Schwarz. Conforme destacado por Tyszler (2006) apud Almeida (2012), o melhor modelo é aquele que apresenta o menor valor do critério de informação. Assim, o SDM apresentou-se o mais adequado para todas as estimativas (Apêndice A).

Nesse modelo, todas as variáveis contidas na matriz X (variáveis explicativas) podem transbordar espacialmente. Para isso, é incluído um componente de transbordamento espacial WX. Ademais, acrescenta-se a variável dependente defasada espacialmente (Wy), tendo (9).

$$
\mathbf{y}=\rho W y+X \pi_{1}+W X \pi_{2}+\varepsilon
$$

Ao estimar $(8)^{3}$, fez-se os testes para heterocedasticidade (Breusch-Pagan) e para a normalidade dos resíduos (Jarque-Bera). Como detectou-se tais problemas econométricos (Apêndice B), aplicou-se os Erros Robustos de White para a correção da heterocedasticidade e para a não normalidade dos erros foram estimados os modelos espaciais pelo Método Generalizado dos Momentos (GMM).

Como o Brasil apresenta uma heterogeneidade na distribuição dos seus recursos produtivos, fez-se estimativas adicionais, usando uma variável categórica (discreta) para identificar regimes espaciais, tendo dois grupos: Sul/Sudeste/Centro-Oeste e Norte/Nordeste. As evidências empíricas apresentadas na análise mostram as diferentes dinâmicas de crescimento entre essas regiões

\footnotetext{
${ }^{3}$ A matriz de convenção utilizada nas estimativas foi a de seis vizinhos, tendo em vista que foi a que apresentou os maiores resultados na investigação da distribuição espacial do resíduo quando estimado por MQO.
} 
(heterogeneidade espacial), justificando tal procedimento. A estabilidade estrutural do modelo de regimes espaciais foi testada via teste de Chow espacial (Anexo C), e no Apêndice $B$ tem os testes focados do tipo Multiplicador de Lagrange (ML) ratificando o SDM como o modelo mais adequado para as estimativas do regime espacial.

\subsection{Especificação das variáveis e Fonte}

O Modelo de convergência de MRW (8) foi estimado para todos os municípios brasileiros (5565). Como o objetivo era utilizar o menor nível de desagregação espacial ${ }^{4}$, e dado que se teve a criação de vários municípios ao longo dos anos de 200o, utilizou-se os dados do Atlas do Desenvolvimento (IDHlongevidade, IDH-conhecimento, população, renda per capita média), o qual já faz o ajuste espacial, com informações de 2000 e $2010^{5}$. Além dessa fonte, coletou-se dados da Rais (estabelecimentos industriais e escolaridade dos trabalhadores), pegando simultaneamente os anos de 2002 e 2010, procedimento que também permite o ajuste espacial.

A taxa de crescimento da renda média entre 2000 e $2010^{6}$ foi utilizada como variável dependente de (8). Como explicativas, considerou os estabelecimentos industriais per capita como proxy para o capital físico (conforme Carmo et al, 2016), e a taxa de crescimento populacional entre 2000 e 2010. Para o capital humano, duas proxies foram utilizadas: escolaridade média do trabalhador (QT), representando a quantidade de capital humano, e; a QT ponderado pela média do IDH-conhecimento e IDH-longevidade, correspondendo a qualidade do capital humano $(\mathrm{QL})^{7}$. A construção de $\mathrm{QL}$ seguiu parcialmente a metodologia de Nakabashi e Figueredo (2005). Esses autores ponderaram a escolaridade pelo IDH, com a hipótese de que melhores condições de vida, incluindo aspectos sociais e educacionais, tenderiam a resultar indivíduos com maior habilidade, com produtividade mais elevada. No entanto, uma das dimensões do IDH é a renda per

\footnotetext{
${ }^{4}$ Isso porque trabalhos como o de Resende et al. (2014) apontam que os determinantes do crescimento econômico podem divergir dependendo da escala geográfica que se usa, inferindo que quanto menor a desagregação, mais preciso é o resultado.

${ }_{5}^{5}$ De acordo com o Atlas do Desenvolvimento (2013), os dados disponibilizados levam em conta a "criação de novos municípios devido às divisões municipais ocorridas entre 2000 ( 5.507 municípios) e 2010 (5.565 municípios) e reproduz o espaço atual dos novos municípios no passado, embora esse espaço não fosse um município e, sim, parte de um ou mais municípios. Dessa forma, é mudado também o espaço daqueles municípios que perderam território. Ele é visto diante da configuração municipal do presente e não do passado".

${ }^{6}$ A renda per capita média de cada município que está disponibilizada no Atlas já vem deflacionada, com $2010=100$.

${ }^{7} \mathrm{O}$ cálculo de QL ficou:

$Q L=Q T \times[(I D H$ longevidade $+I D H$ conhecimento $) / 2]$
} 
capita. Como a variável dependente de (8) refere-se à taxa de crescimento da renda per capita, optou-se por ponderar QT apenas pela dimensão longevidade e conhecimento do IDH, visando evitar correlações supervalorizadas entre QL e a variável dependente.

Isso posto, duas estimativas foram feitas usando o modelo Durbin espacial: na primeira a proxy para o capital humano foi o QT (10), e na segunda, a proxy foi o QL (11).

$$
\begin{aligned}
& \ln \left(\frac{Y_{t m}}{Y_{0 m}}\right)=b_{0}+b_{1} \ln K F_{0 m}+b_{2} \ln Q T_{0 m}+b_{3} \ln \text { txpop }_{m}+b_{4} \ln Y_{0 m}+b_{5} W \ln K F_{0 m}+ \\
& b_{6} W \ln Q T_{0 m}+b_{7} W \ln \text { txpop }_{m}+b_{8} W \ln Y_{0 m}+\rho W\left[\ln \left(\frac{Y_{t m}}{Y_{0 m}}\right)\right]+\varepsilon \\
& \ln \left(\frac{Y_{t m}}{Y_{0 m}}\right)=b_{0}+b_{1} \ln K F_{0 m}+b_{2} \ln Q L_{0 m}+b_{3} \ln \text { txpop }_{m}+b_{4} \ln Y_{0 m}+b_{5} W \ln K F_{0 m}+ \\
& b_{6} W \ln Q T_{0 m}+b_{7} W \ln \text { txpop }_{m}+b_{8} W \ln Y_{0 m}+\rho W\left[\ln \left(\frac{Y_{t m}}{Y_{0 m}}\right)\right]+\varepsilon
\end{aligned}
$$

Em que: $\mathbf{Y}$ é a renda per capita média; KF é o capital físico (proxy: estabelecimentos industriais per capita); Qt é a quantidade de capital humano; QL é a qualidade do capital humano; txpop é o crescimento da produtividade entre 2000 e 2010; $r$ refere-se ao coeficiente espacial da defasagem da variável dependente; $\mathbf{t}$ é o ano final (2010), e; o é o ano inicial (2000 ou 2002, dependendo da base de dados).

Foi considerado os dados do capital físico e do capital humano do ano inicial com o objetivo de captar seu efeito subsequente (2000/2010) no crescimento da renda per capita. Ademais, ao realizar tal procedimento, evita-se parcialmente efeitos de endogeneidade.

Como o Brasil apresenta uma heterogeneidade na distribuição dos seus recursos produtivos, os modelos (10) e (11) foram reestimados, usando uma variável categórica (discreta) para identificar regimes espaciais Sul/Sudeste/Centro-Oeste versus Norte/Nordeste. Separou-se os municípios nesses dois grupos tendo em vista as diferenças nas dinâmicas econômicas (heterogeneidade espacial) do Norte/Nordeste frente o restante do país. 
3. Capital humano e seu impacto nos municípios brasileiros: evidências empíricas

Entender a dinâmica do crescimento econômico é imprescindível para identificar tendências, bem como para rever processo negativos ou estagnados. Quando o foco é uma região heterogênea, como é o caso brasileiro, análises macros podem camuflar os resultados negativos que regionalmente se tem. Portanto, as investigações regionais, observadas nos menores níveis de desagregação, apresentam-se como importantes ferramentas para se ter processos de crescimento econômico mais equilibrado.

O contexto da análise regional paira numa busca visando identificar os determinantes desse crescimento econômico. A partir da década de 1950, autores como Mincer (1958), Becker (1964) e Shultz (1963) apontaram para a existência de um importante fator que até aquele momento estaria sendo negligenciado nas análises econômicas, referindo-se ao capital humano. Ignorar essa variável significa supor que o trabalho é homogêneo, podendo ser facilmente substituível. Entretanto, esses autores pioneiros evidenciaram que pessoas mais qualificadas, com maior treinamento formal, com qualidade maior de saúde e bem-estar, etc., podem diferenciar suas habilidades, suas produtividades, impactando nas suas remunerações, bem como no nível de produtividade regional. A partir desses e de outros argumentos, o capital humano passou a ser incorporado nos modelos de crescimento, como o de Romer (1986), Lucas (1988), revelando um impacto positivo nesse processo.

A nível regional, alguns trabalhos, como Connolly (2004) e Nakabashi e Salvato (2007), comprovaram essa importância do capital humano para o dinamismo econômico. Como a mobilidade da mão de obra, referindo-se especialmente aos indivíduos mais produtivos e com maior habilidade, tende a ser mais intensa internamente no país do que entre países, a dinâmica de sua localização e de sua movimentação pode estar determinando o desempenho regional brasileiro.

Por isso, analisou-se a dinâmica de distribuição e de evolução do capital humano. Na Tabela 1 é apresentado esses resultados para os municípios do Brasil. Observa-se uma evolução importante, com o crescimento dos valores médios tanto do QT (quantidade de capital humano) como do QL (qualidade do capital humano), além do aumento dos valores mínimos e máximos. Soma-se a isso a existência de uma distribuição mais homogênea dessas variáveis, com uma diminuição expressiva do coeficiente de variação. Isso significa que está se tendo uma maior formação do capital humano, tanto em termos quantitativos como qualitativos, e estes estão se distribuindo de uma forma mais igualitária. É claro que ainda persiste uma discrepância significativa no que se refere aos valores do 
QL e do QT entre os municípios, contudo, essa diferença vem sendo reduzida com o passar dos anos.

Tabela 1: Estatística descritiva do capital humano - municípios brasileiros em 2000 e 2010

\begin{tabular}{cccccc}
\hline Capital humano & Média & Máximo & Mínimo & Desvio padrão & Coeficiente de variação \\
\hline QT 2000 & 8,43 & 14,64 & 2,50 & 1,25 & 14,82 \\
QT 2010 & 9,87 & 15,00 & 6,08 & 1,02 & 10,31 \\
QL 2000 & 4,44 & 11,79 & 0,31 & 1,11 & 25,05 \\
QL 2010 & 6,71 & 12,89 & 2,00 & 0,97 & 14,50 \\
\hline
\end{tabular}

Fonte: Resultado da pesquisa.

Nota: QT é a quantidade de capital humano (escolaridade média do trabalhador); QL é a qualidade do capital humano, correspondendo à QT x [(IDH longevidade + IDH conhecimento)/2].

Visualmente, esses resultados podem ser comprovados pela Figura 1, com uma inserção maior de municípios nas duas melhores escalas do QL e do QT, quando comparado 2000 e 2010.

Analisando o padrão de distribuição espacial das proxies do capital humano, constata-se a não aleatoriedade de ambas (Tabela 2 ), de modo que, independente da convenção que se utilize, o coeficiente I de Moran ${ }^{8}$ é positivo e estatisticamente significativo. Ou seja, aqueles municípios com elevado QL ou QT tendem a ter no seu envoltório municípios que também detém altos valores (e vice-versa). Assim, embora praticamente todos os municípios elevaram a quantidade (97\% dos municípios) e a qualidade do capital humano (99\%), persiste um padrão de distribuição dos melhores resultados, concentrando-os espacialmente, assim como também se tem um padrão de concentração dos piores resultados.

Comparando a qualidade versus a quantidade de capital humano distribuído ao longo do país, tem-se uma concentração maior da qualidade no Sul/Sudeste, enquanto que para a quantidade tem-se uma centralização mais elevada dos melhores valores no Nordeste. Ao mesmo tempo em que se tem essa dispersão de localização dos melhores resultados, ao fazer uma correlação entre todos os valores do QL e do QT foi encontrado um coeficiente positivo e estatisticamente significativo (o,70 para 2000 e 0,73 para 2010), o que significa que parte dos municípios que detém uma boa quantidade de capital humano tem um valor alto da qualidade.

\footnotetext{
${ }^{8}$ A estatística I de Moran permite identificar se a distribuição espacial de uma determinada variável ocorre de forma aleatória ou não. A hipótese nula a ser testada é a de que a distribuição espacial da variável é aleatória. (ALMEIDA, 2012).
} 
A questão a ser investigada é o quanto essas duas proxies do capital humano interferem na dinâmica do crescimento da renda per capita do país.

Figura 1: Distribuição da Qualidade (QL) e da quantidade do capital humano (QT) - municípios brasileiros - 2000 e 2010

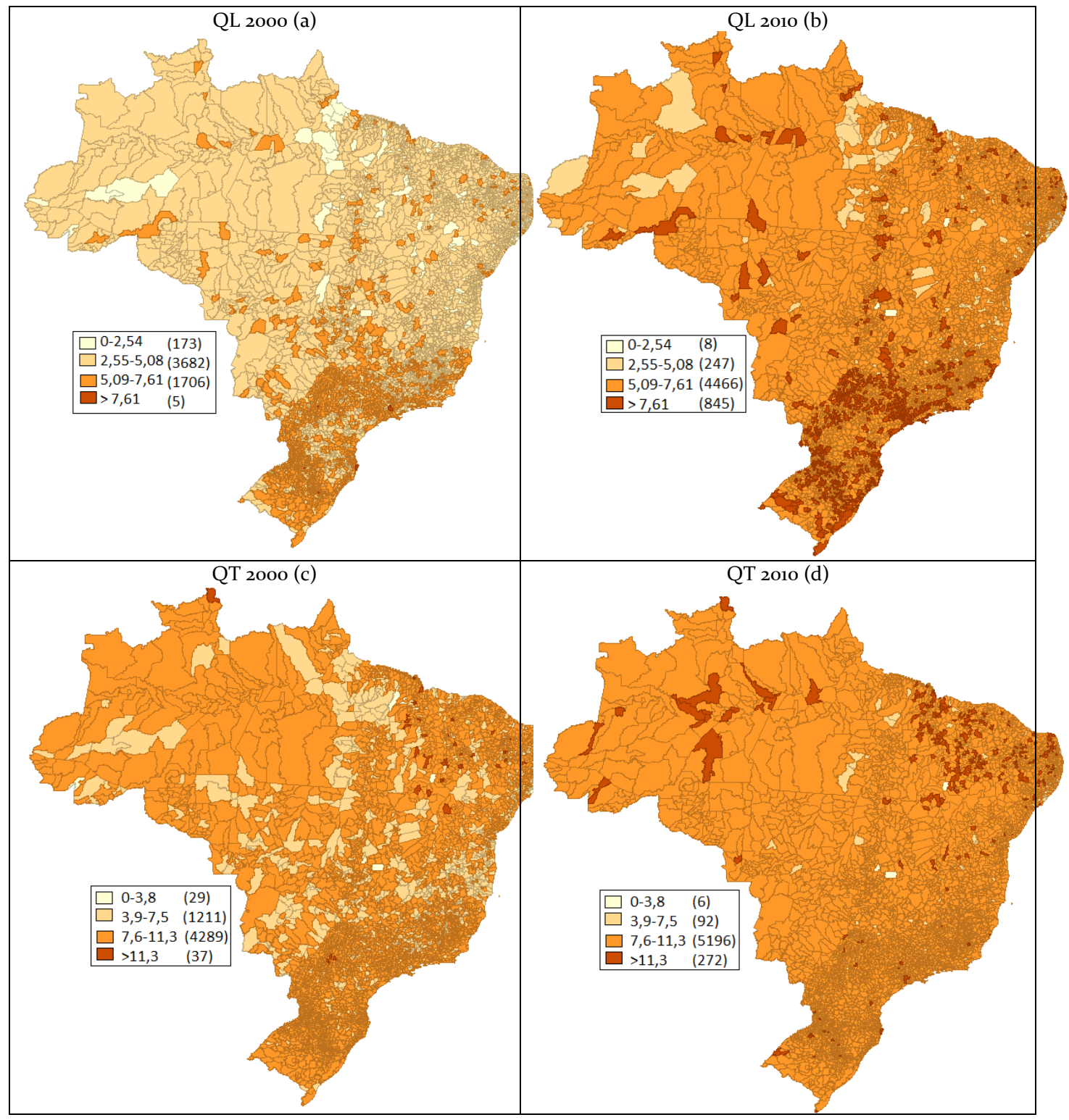

Fonte: Resultado da pesquisa 
Tabela 2: Estatística I de Moran - capital humano - municípios brasileiros em 2000 e 2010

\begin{tabular}{ccccc}
\hline Capital Humano & \multicolumn{3}{c}{ Convenção } \\
\cline { 2 - 5 } & Rainha & Torre & 4 vizinhos & 5 vizinhos \\
\hline QT 2000 & $0,27^{*}$ & $0,27^{*}$ & $0,29^{*}$ & $0,29^{*}$ \\
QT 2010 & $0,29^{*}$ & $0,30^{*}$ & $0,31^{*}$ & $0,29^{*}$ \\
QL 2000 & $0,58^{*}$ & $0,58^{*}$ & $0,58^{*}$ & $0,58^{*}$ \\
QL 2010 & $0,45^{*}$ & $0,45^{*}$ & $0,45^{*}$ & $0,45^{*}$ \\
\hline
\end{tabular}

Fonte: Estimado pelos autores por meio do software Geoda.

Nota: A pseudo significância empírica baseada em 999 permutações aleatórias; * significativo a um nível de significância de $5 \%$.

Analisando a distribuição da renda per capita entre os municípios brasileiros (Figura 2), teve-se resultados positivos, com intensificação dos valores médios, atrelado à redução das discrepâncias. Ao mesmo tempo, verificou-se uma alta heterogeneidade no que se refere à taxa de crescimento da renda per capita, chegando a ter municípios com um crescimento de 530\%, enquanto outros decresceram. Dois pontos devem ser destacados: 1) os que não conseguiram elevar sua renda per capita somaram apenas 3\% dos municípios brasileiros, e; 2) essa diferença de crescimento pode estar se dando em favor dos municípios que detinham uma renda per capita inicial menor, o que conduziria a um processo de convergência de renda ao longo do país.

Com efeito, essa hipótese é reforçada quando se compara a Figura 2a com a Figura 2c, em que parte dos municípios com baixa renda per capita em 2000 tiveram ritmos de crescimento mais intenso na sequência.

No que se refere à distribuição espacial, tem-se um padrão bem definindo, centrando-se os melhores resultados nas regiões Sul/Sudeste/Centro-Oeste do país. E esse padrão de distribuição, que não é aleatório espacialmente (conforme estatística I de Moran, Tabela 4), intensificou-se com o passar dos anos, constatação observada visualmente ao comparar as Figuras $2 \mathrm{a}$ e $2 \mathrm{~b}$ e corroborado pelo valor do coeficiente I de Moran, maior em 2010 do que em 2000.

Tabela 3: Estatística descritiva - Renda per capita e taxa de crescimento - municípios brasileiros em 2000 e 2010

\begin{tabular}{cccccc}
\hline \multirow{2}{*}{ Renda per capita } & Média & Máximo & Mínimo & Desvio padrão & Coeficiente de variação \\
\hline 2000 & 308,58 & $1.759,76$ & 62,65 & 192,46 & 62,37 \\
2010 & 467,65 & $2.043,74$ & 96,25 & 243,29 & 52,02 \\
Taxa de cresc. 2000/2010 & 51,40 & 530,33 & $-32,23$ & 33,93 & 66,01 \\
\hline
\end{tabular}

Fonte: Resultado da pesquisa 
Tabela 4: Estatística I de Moran - renda per capita - municípios brasileiros de 2000 e 2010

\begin{tabular}{c|c|c|c|c}
\hline \multirow{2}{*}{ Renda per capita } & \multicolumn{3}{c}{ Convenção } \\
\cline { 2 - 5 } & Rainha & Torre & 4 vizinhos & 5 vizinhos \\
\hline 2000 & $0,68^{*}$ & $0,67^{*}$ & $0,66^{*}$ & $0,66^{*}$ \\
2010 & $0,70^{*}$ & $0,70^{*}$ & $0,70^{*}$ & $0,70^{*}$ \\
\hline
\end{tabular}

Fonte: Estimado pelos autores por meio do software Geoda.

Nota: A pseudo significância empírica baseada em 999 permutações aleatórias; * significativo a um nível de significância de 5\%. 
Figura 2: Renda per capita e da taxa de crescimento - municípios brasileiros em 2000 e 2010.

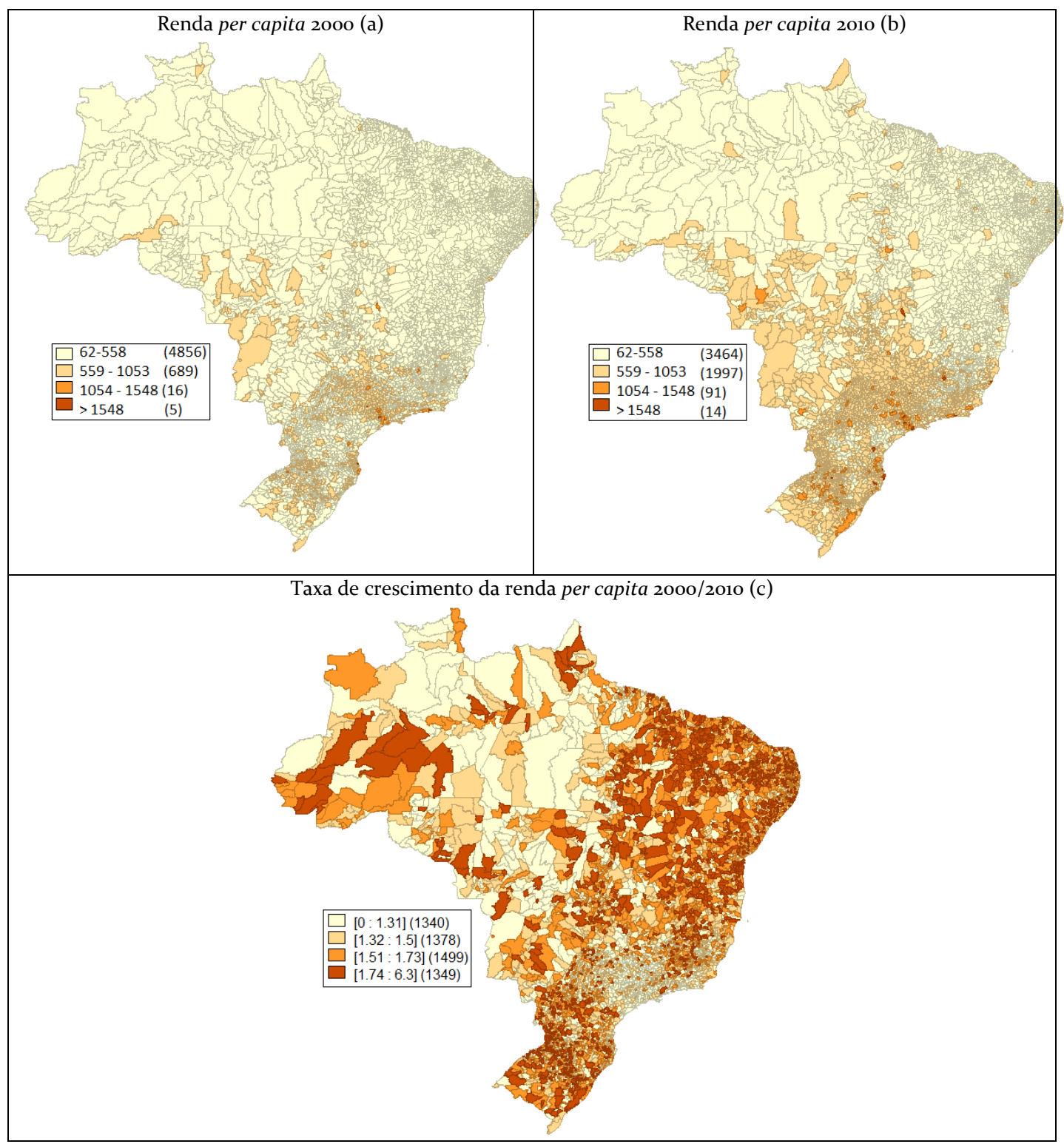

Fonte: Resultado da pesquisa 
Ademais, na Figura $3^{9}$ reforça-se esse argumento, tendo uma fomentação da concentração espacial dos grupos de municípios com altos valores da renda per capita cercados por municípios com elevados montantes (alto-alto), e daqueles grupos de municípios com baixos valores cercados por municípios com situação similar (baixo-baixo), evidenciando também a desigualdade na localização desses clusters, ficando preponderantemente o grupo alto-alto no Sul/Sudeste/CentroOeste e o baixo-baixo no Nordeste/Norte.

\footnotetext{
9 Por meio da auto correlação local (testada por meio da estatística I de Moran Local) é possível obter um indicador para cada município em análise e, com isso, identificar a presença de clusters (agrupamentos de valores similares) retratado no mapa de cluster do tipo LISA (Local Indicator of Spatial Association). Cada município possuirá a sua estatística I de Moran e seu respectivo nível de significância, e aqueles municípios com valores estatisticamente significativos compõe cada clusters.
} 
Figura 3: Mapa Lisa - Renda per capita e taxa de crescimento 2000/2010 - municípios brasileiros

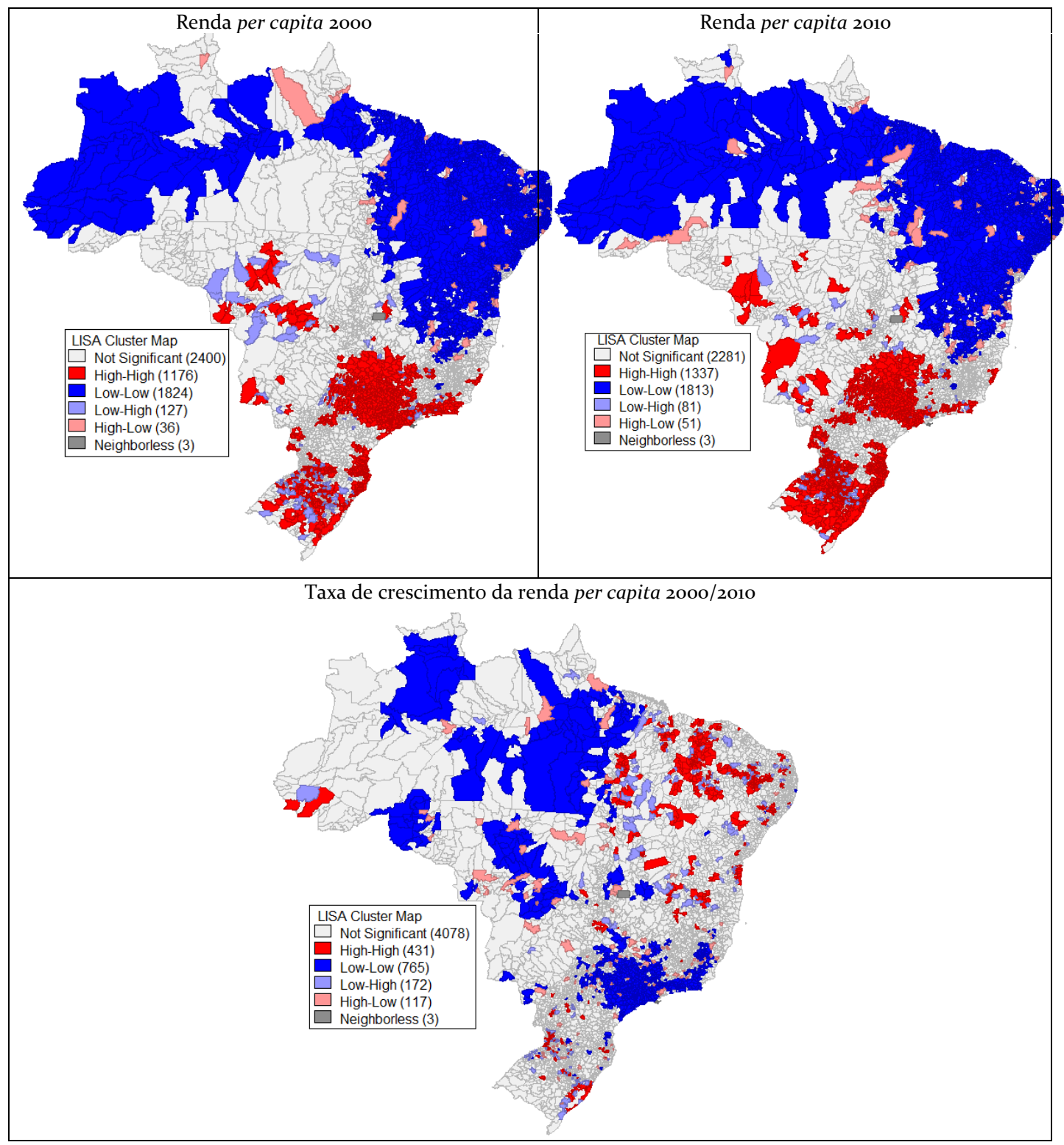

Fonte: Geoda 
Isso posto, questiona-se quais os fatores que estariam atuando nessa dinâmica, e qual a relevância do capital humano nesse processo. Além disso, alguns trabalhos, como Hanushek e Kimko (2000) e Nakabashi e Figueiredo (2005) demonstram que mais importante que a quantidade de capital humano existente numa região é a sua qualidade. Será que isso é verificado no caso dos municípios brasileiros? Será que o capital humano tem impacto no processo de geração de renda entre os municípios do país? Os resultados das estimativas (10) e (11) são apresentados na Tabela $5 \mathrm{com}$ o intuito de responder esses e outros questionamentos.

Como corolário, em ambos os modelos está se evidenciando um processo de convergência de renda, com intensidade maior quando a proxy para o capital humano se refere à qualidade, de modo que aqueles municípios com renda per capita inicial menor apresentaram, na média, um crescimento econômico subsequente maior. Essa inferência ratifica a dinâmica que se observou nas Figura 2a e 2c. Assim, por mais que hoje o dinamismo econômico ainda esteja concentrado espacialmente, existe um processo de homogeneização, o qual tende, no longo prazo, diminuir as discrepâncias existentes ao longo do país, ceteris de paribus.

Entretanto, quando se defasou espacialmente a variável "renda inicial" se obteve um coeficiente positivo e estatisticamente significativo. Isso significa que municípios que tinham inicialmente um dinamismo maior apresentaram no seu envoltório um crescimento econômico mais intenso, da mesma forma que aqueles que apresentavam inicialmente um processo econômico mais desfavorável, na média, tenderam a ter na sua vizinhança municípios com acanhado crescimento da renda per capita. Isso justifica especialmente a ampliação do número de municípios do cluster alto-alto da Figura 3.

Resumidamente, no caso dos municípios com renda per capita inicial alta, apresentaram dois fenômenos: na média, obtiveram um crescimento econômico subsequente menor, e; tenderam a ter no seu envoltório municípios com taxas de crescimento da renda per capita mais intensas. Nesse contexto, pode-se inferir que o processo de convergência está se dando especialmente naquelas regiões nas quais já se tinham algum foco de dinamismo econômico inicial, sinalizando para um processo de convergência de renda desigual regionalmente.

No caso da variável "capital físico", apresentou um efeito positivo e estatisticamente significativo tanto no modelo (10) como em (11). A sua defasagem espacial indica um efeito negativo na vizinhança. Ora, autores como Hirschmann (1958) destacam que muitas vezes um aglomerado produtivo pode gerar impactos negativos para a sua vizinhança por meio de vazamentos, por exemplo, da mão de obra mais especializada, dentre outros fatores. Assim, é possível identificar tal fenômeno entre os municípios do Brasil. 
O parâmetro $r$ exibiu impacto positivo e significativo em ambas as estimativas, demonstrando um transbordamento positivo do crescimento da renda per capita na dinâmica econômica dos municípios do envoltório. Isso mostra que, quando determinado município cresce, parte desse crescimento econômico também acaba beneficiando os seus vizinhos, criando um ciclo virtuoso do crescimento.

Focando no capital humano, o modelo (10) revela um impacto positivo e estatisticamente significativo para a quantidade de capital humano. Portanto, municípios com escolaridades médias maiores dos seus trabalhadores tendem a ter um crescimento subsequente da renda per capita mais intenso, mostrando a importância desse fator para o processo econômico dos municípios brasileiros. Da mesma maneira, a qualidade do capital humano - modelo (11) - também apresentou um efeito positivo, e seu impacto no processo de crescimento econômico tende a ser maior, dado que o seu coeficiente é superior ao obtido para o QT.

Cabe ressaltar que esses resultados contradizem, em parte, o processo de convergência de renda que foi visualizado com o coeficiente negativo da renda per capita inicial, dado que, quando se tem um maior nível de capital humano tem-se, na sequência, um incremento ainda maior da renda per capita e, como o capital humano em geral localiza-se especialmente em regiões com níveis de renda alto, isso pode frear ou inibir o processo de convergência. Como solução, se políticas públicas forem efetivadas visando homogeneizar o capital humano em todo o espaço brasileiro, com ênfase maior naqueles municípios com dinamismo econômico menor, ter-se-ia, então, um processo de intensificação do fenômeno de convergência derivado dessa melhor distribuição do capital humano ao longo do país.

Quando defasada espacialmente as duas proxies do capital humano, ambas obtiveram um coeficiente negativo e estatisticamente significativo. Assim, o efeito do capital humano (qualidade e quantidade) é positivo no município que está concentrando-o, contudo, afeta negativamente a dinâmica econômica dos municípios vizinhos. Krugman (1990) infere que forças centrípetas atuam atraindo o fator trabalho para espaços de concentração produtiva, especialmente pelas oportunidades de emprego, pelos salários maiores oriundos da demanda maior por mão de obra, pelo maior poder de barganha que o trabalhador adquire ao se localizar onde já se tem uma concentração desse fator produtivo. Portanto, quando um município eleva o seu estoque de capital humano (em termos qualitativo e/ou quantitativo), outros trabalhadores qualificados tendem a se deslocar do envoltório, reduzindo indiretamente a produtividade da vizinhança e afetando negativamente o seu ritmo de crescimento econômico. Essa seria uma das possíveis explicações para o efeito negativo do capital humano nos municípios vizinhos. 
Tabela 5: Resultado das estimativas econométrica dos modelos (10) e (11) - municípios brasileiros

\begin{tabular}{ccc}
\hline & & Modelo \\
\cline { 2 - 3 } & $(10)$ & $(11)$ \\
\hline Constante & $-1,21^{*}$ & $-1,09^{*}$ \\
& $(0,41)$ & $(0,36)$ \\
Ln KF & $0,03^{*}$ & $0,02^{*}$ \\
& $(0,01)$ & $(0,003)$ \\
Ln txpop & $-0,02$ & $-0,02$ \\
& $(0,02)$ & $(0,02)$ \\
Ln QL & - & $0,20^{*}$ \\
& Ln QT & $(0,01)$ \\
& $0,16^{*}$ & - \\
Ln $Y_{o}$ & $(0,03)$ & $-0,38^{*}$ \\
$\rho$ & $-0,35^{*}$ & $(0,01)$ \\
& $(0,04)$ & $1,45^{*}$ \\
W Ln KF & $1,64^{*}$ & $(0,14)$ \\
& $(0,21)$ & $-0,06^{*}$ \\
W Ln txpop. & $-0,09^{*}$ & $(0,01)$ \\
& $(0,02)$ & 0,10 \\
W Ln QL & $0,13^{*}$ & $(0,14)$ \\
W Ln QT & $(0,05)$ & $-0,34^{*}$ \\
& - & $(0,07)$ \\
W Ln $Y_{0}$ & $-0,39^{*}$ & - \\
& $(0,07)$ & $0,54^{*}$ \\
& $0,55^{*}$ & $(0,06)$ \\
\hline
\end{tabular}

Fonte: GeodaSpace.

Nota: Y é a renda per capita média; KF é o capital físico (proxy: estabelecimentos industriais per capita); Qt é a quantidade de capital humano; QL é a qualidade do capital humano; txpop é o crescimento da produtividade entre 2000 e 2010; $r$ coeficiente espacial da defasagem da variável dependente; $\mathbf{t}$ refere-se ao ano final (2010), e; o é o ano inicial (2000 ou 2002, dependendo da base de dados); Ln é logaritmo; * nível de significância de $1 \%$.

Embora se tenha identificado uma tendência geral de convergência de renda entre os municípios brasileiros, com importantes efeitos do capital humano nesse processo, existe uma heterogeneidade na distribuição da dinâmica econômica e dos recursos produtivos entre os municípios do país (Figura 1 e 2). Conforme destacam Galor e Zeira (1993), as rendas per capita das regiões que possuem características estruturais iniciais próximas tendem a convergir para o mesmo ponto, existindo, portanto, múltiplos equilíbrios estáveis, e não uma única convergência para todas as regiões.

Os autores explicam como a distribuição inicial da riqueza influencia, por exemplo, na decisão de investimento em capital humano dos indivíduos, 
argumentando que se o custo de financiamento do investimento em capital humano não é o mesmo entre grupos de indivíduos com distribuição inicial de riqueza diferente, o retorno desse investimento também será desigual em tais grupos. Nesse sentido, o indivíduo que não possuir um determinado valor de riqueza inicial pode preferir não investir em educação e trabalhar como mão de obra não qualificada. Como os salários e os ganhos futuros dependem do capital humano que o indivíduo possui, ter-se-ão regiões com trabalhadores qualificados ganhando melhores salários do que outras regiões com trabalhadores não qualificados, destacando que essa diferença tende a ser maior quanto mais elevado for a desigualdade inicial na distribuição da riqueza. É nesse sentido que a distribuição inicial da renda influencia na desigualdade de renda no longo prazo.

No caso do Brasil, na Tabela 6 tem-se a renda per capita média para as regiões brasileiras. Os dados revelam diferenças entre os valores do Norte/Nordeste e das demais regiões do país, podendo chegar até R\$427 em 2010 e $\mathrm{R} \$ 290$ em 2000.

Considerando essa heterogeneidade é que se analisou a possibilidade de convergência em clube, reestimando (10) e (11), com resultados para os municípios do Norte/Nordeste (No/Nor) e outro para Sul/Sudeste/Centro-Oeste (SU/SE/CO) (Tabela 7), por meio de modelos de regimes espaciais.

Tabela 6: Renda per capita média, regiões brasileiras em 2000 e 2010

\begin{tabular}{ccc}
\hline & Renda média per capita 2000 & Renda média per capita 2010 \\
\hline Norte & 242,4 & 345,1 \\
Nordeste & 170,0 & 277,0 \\
Sudeste & 438,6 & 590,6 \\
Sul & 460,8 & 704,2 \\
Centro-Oeste & 410,4 & 586,6 \\
\hline
\end{tabular}

Fonte: Resultado da pesquisa

Como corolário, independente do modelo estimado (10) ou (11), tem-se um processo de convergência para ambos os grupos de municípios. No entanto, o processo é mais intenso para o grupo No/Nor, especialmente quando a proxy para o capital humano é a qualidade. Como esse grupo apresenta um nível de dinamismo econômico inicial menor, é compreensível se ter um processo de fomentação da renda maior. A contradição está no coeficiente da defasagem espacial do $Y_{o}$, não apresentando significância estatística para esse grupo, não 
tendo efeito de transbordamento sobre o crescimento da renda per capita dos municípios vizinhos.

No caso do SU/SE/CO, tem-se um processo de convergência, o qual também é superior quando a proxy para o capital humano é a qualidade, além do que naqueles municípios com renda per capita inicial alta tem-se, na média, municípios com superiores taxas de crescimento da renda na sua vizinhança.

Assim, o processo de convergência da renda do No/Nord parece ser mais desconcentrado do que a do grupo SU/SE/CO, em que os transbordamentos de municípios desenvolvidos, na média, afetam o dinamismo do envoltório, reforçando a formação dos clusters alto-alto.

No caso do efeito do capital humano na taxa de crescimento da renda, para ambos os grupos, constatou-se um impacto positivo independente da proxy utilizada (QT e QL), ressaltando que a qualidade do capital humano apresentou superioridade de efeito em ambos os grupos. A mesma ressalva se faz quanto à inibição do processo de convergência de renda per capita que tal resultado pode estar sinalizando, dado que, o crescimento econômico tenderia a ser mais forte nos municípios que teriam a formação inicial de capital humano maior. Por isso, devese atentar a tal fato, com políticas focadas visando diminuir os gargalos dessa distribuição da qualidade e da quantidade de capital humano em todas as regiões brasileiras.

Quando comparado o coeficiente de QT e de QL para o SU/SE/CO versus o No/Nord, tem-se um valor maior para o primeiro. Ou seja, uma capacitação maior, uma escolaridade maior, um nível de bem-estar superior afeta mais fortemente a taxa de crescimento da renda do SU/SE/CO do que do No/Nor. Talvez esse fato esteja relacionado com a estrutura produtiva, mais intensa em tecnologia na região $\mathrm{SU} / \mathrm{SE} / \mathrm{CO}$, e consequentemente apresentando uma maior demanda por mão de obra qualificada.

Por fim, na análise do efeito de transbordamento do capital humano para o dinamismo econômico do envoltório, apenas a qualidade apresentou um efeito, com impacto negativo similar ao observado para a regressão de todos os municípios (Tabela 5), e esse efeito só foi significativo para o SU/SE/CO. 
Tabela 7: Resultado das estimativas econométrica dos modelos (10) e (11) para grupos de municípios - regiões brasileiras Modelo

\begin{tabular}{|c|c|c|c|c|}
\hline \multirow[t]{2}{*}{ Variável } & \multicolumn{2}{|c|}{ (10) } & \multicolumn{2}{|c|}{ (11) } \\
\hline & No/Nor & $\mathrm{SU} / \mathrm{SE} / \mathrm{CO}$ & No/Nor & $\mathrm{SU} / \mathrm{SE} / \mathrm{CO}$ \\
\hline Constante & $\begin{array}{l}2,42^{*} \\
(0,61)\end{array}$ & $\begin{array}{c}0,02 \\
(0,94)\end{array}$ & $\begin{array}{c}2,19^{*} \\
(0,68)\end{array}$ & $\begin{array}{c}0,18 \\
(0,33)\end{array}$ \\
\hline Ln $\mathrm{KF}_{\mathrm{o}}$ & $\begin{array}{l}\mathrm{o}, 04^{*} \\
(\mathrm{o}, \mathrm{O} 1)\end{array}$ & $\begin{array}{l}\mathrm{O}, 02^{*} \\
(\mathrm{o}, \mathrm{O} 1)\end{array}$ & $\begin{array}{c}0,04^{*} \\
(0,006)\end{array}$ & $\begin{array}{l}0,004^{*} \\
(0,002)\end{array}$ \\
\hline Ln txpop & $\begin{array}{l}-0,06 \\
(0,03)\end{array}$ & $\begin{array}{l}-0,02 \\
(0,03)\end{array}$ & $\begin{array}{c}-0,06^{* *} \\
(0,03)\end{array}$ & $\begin{array}{c}-0,008 \\
(0,03)\end{array}$ \\
\hline Ln $\mathrm{QL}_{\mathrm{o}}$ & - & - & $\begin{array}{c}0,13^{*} \\
(0,02)\end{array}$ & $\begin{array}{l}0,44^{*} \\
(0,02)\end{array}$ \\
\hline $\mathrm{Ln}_{\mathrm{QT}}$ & $\begin{array}{l}0,07^{*} \\
(\mathrm{o}, 07)\end{array}$ & $\begin{array}{l}0,40^{*} \\
(0,05)\end{array}$ & - & - \\
\hline $\operatorname{Ln} Y_{0}$ & $\begin{array}{c}-0,40^{*} \\
(0,02)\end{array}$ & $\begin{array}{l}-0,34^{*} \\
(0,04)\end{array}$ & $\begin{array}{l}-0,43^{*} \\
(0,02)\end{array}$ & $\begin{array}{c}-0,36 \\
(0,06)\end{array}$ \\
\hline$\rho$ & $\begin{array}{c}0,21 \\
(0,19)\end{array}$ & $\begin{array}{c}1,0^{*} \\
(0,14)\end{array}$ & $\begin{array}{l}0,34^{* *} \\
(0,20)\end{array}$ & $\begin{array}{l}0,94^{*} \\
(0,13)\end{array}$ \\
\hline $\mathrm{W}$ Ln $\mathrm{KF}_{\mathrm{o}}$ & $\begin{array}{c}0,04 \\
(0,02)\end{array}$ & $\begin{array}{l}-0,34^{*} \\
(0,04)\end{array}$ & $\begin{array}{c}0,03 \\
(0,25)\end{array}$ & $\begin{array}{c}-0,008 \\
(0,02)\end{array}$ \\
\hline W Ln txpop. & $\begin{array}{c}0,04 \\
(0,04)\end{array}$ & $\begin{array}{c}0,02 \\
(0,04)\end{array}$ & $\begin{array}{c}0,03 \\
(0,04)\end{array}$ & $\begin{array}{c}0,02 \\
(0,05)\end{array}$ \\
\hline $\mathrm{W} \operatorname{Ln} \mathrm{QL}_{\mathrm{o}}$ & - & - & $\begin{array}{c}-0,02 \\
(0,04)\end{array}$ & $\begin{array}{l}-0,30^{*} \\
(0,07)\end{array}$ \\
\hline $\mathrm{W} \operatorname{Ln} \mathrm{QT}_{\mathrm{o}}$ & $\begin{array}{c}0,07 \\
(0,04)\end{array}$ & $\begin{array}{c}-0,40^{*} \\
(0,1)\end{array}$ & - & - \\
\hline$W \operatorname{Ln} Y_{o}$ & $\begin{array}{c}0,04 \\
(0,09)\end{array}$ & $\begin{array}{l}0,34^{*} \\
(0,07)\end{array}$ & $\begin{array}{l}0,11 \\
(0,1) \\
\end{array}$ & $\begin{array}{l}0,32^{*} \\
(0,00)\end{array}$ \\
\hline
\end{tabular}

Fonte: GeodaSpace.

Nota: Y é a renda per capita média; KF é o capital físico (proxy: estabelecimentos industriais per capita); Qt é a quantidade de capital humano; QL é a qualidade do capital humano; txpop é o crescimento da produtividade entre 2000 e 2010; $r$ coeficiente espacial da defasagem da variável dependente; $\mathbf{t}$ refere-se ao ano final (2010), e; o é o ano inicial (2000 ou 2002, dependendo da base de dados); Ln é logaritmo; * nível de significância de 1\%.

\section{Considerações finais}

O objetivo deste artigo consistiu na identificação do efeito do capital humano - tanto quantitativo como qualitativo - no processo de crescimento da renda per capita dos municípios brasileiros. Os resultados apontaram para a existência de um processo de convergência, o qual é maior quando se tem a qualidade como medida do capital humano. Entretanto, também se identificou que a dinâmica do crescimento econômico tende a ser mais intensa quando se tem um estoque mais elevado de aspectos qualitativo e quantitativo do capital humano. Dado que existe uma disparidade na distribuição do capital humano, 
especialmente da qualidade, se localizando fundamentalmente nos municípios com maior dinamismo econômico, isso pode inibir o processo de convergência de renda se políticas públicas não forem efetivadas, com o intuito de fomentar a formação de capital humano daqueles municípios com menor renda inicial.

Esses mesmos resultados foram evidenciados para grupos de municípios do No/Nord versus SU/SE/CO, ressaltando que neste observou um efeito superior do capital humano na dinâmica da sua renda per capita. Possivelmente isso seja decorrente da localização da estrutura produtiva, a qual está centrada fortemente no SU/SE/CO e, junto dela, tenha-se também uma utilização maior do capital humano disponível.

Ademais, constatou-se, especialmente no SU/SE/CO, que o incremento de QT ou QL de um município pode acarretar em perdas de dinamismo na vizinhança. Esse resultado, atrelado aos demais, sinaliza para a necessidade de se ter políticas públicas dirigidas à formação de capital humano focando especialmente naqueles municípios com uma dinâmica econômica menor, para que não se tenha vazamentos e perdas de desempenho dado o incremento natural de capital humano nos municípios mais dinâmicos.

Tais políticas devem centrar-se não apenas nas metas quantitativas, mas devem primar pela qualidade da formação que está se tendo, não precisando se prender apenas aos aspectos educacionais, mas visar também à formação profissional, à qualidade de vida, ao bem-estar, dentre outros.

É necessário entender a formação do capital humano de cada município, com suas particularidades, e adequar as políticas para que se consiga avançar, melhorando as habilidades, o desempenho individual, e dinamizando a renda interna.

\section{Referências}

ALMEIDA, E. Econometria espacial aplicada. São Paulo: Alínea. 2012.

Atlas do Desenvolvimento Humano. Perguntas frequentes, 2013. Disponível em:< http://www.atlasbrasil.org.br/2013/pt/o atlas/perguntas frequentes/\#15>. Acesso junho, 2017.

BAUMOL, W.J. Productivity growth, convergence, and welfare: what the long-run data show. American Economic Review, v.76, n. 5, p. 1072- 85, 1986.

BARRO, R.J. Education and economic growth. Harvard University, 2000. 
BARRO, R.J.; LEE, J.W. International data on educational attainment: updates and implications. Oxford Economic papers, v. 53, n. 3, p. 541-563, 2001 . DOI: https://doi.org/10.1093/oep/53.3.541

BECKER, G.S. Human capital. New York: NBER, 1964.

BOSWORTH, B.; COLLINS, S.M. The empirics of growth: An update. Brookings papers on economic activity, n.2, p. 113-206, 2003. DOI: https://doi.org/10.1353/eca.2004.0002

CRAWFORD, Richard. Na era do Capital Humano: o talento, a inteligência e o conhecimento como forças econômicas, seu impacto nas empresas e nas decisões de investimento. São Paulo: Atlas, 1994.

CONNOLLY, M.P. Human Capital and Growth in the Post-Bellum South: A Separate but Unequal Story. Journal of Economic History, v. 64, n. 2, p.1-39, 1994.

CARMO, A.S.S.; RAIHER, A.P.; STEGE, A.L. O efeito das exportações no crescimento econômico das microrregiões brasileiras: uma análise espacial com dados em painel. Estud. Econ., v. 47, n.1, p.153-183, 2016. DOI: https://doi.org/10.1590/0101-416147161aaa

FIRME, V. de A.C.; SIMÃO FILHO, J. Análise do crescimento econômico dos municípios de Minas Gerais via modelo MRW(1992) com capital humano, condições de saúde e fatores especiais, 1991-2000. Economia Aplicada, v.18, n. 4, p. 679-706, 2014. DOI: https://doi.org/10.1590/1413-8050/ea640

GALOR, O.; ZEIRA, J. Income distribution and macroeconomics. Review of Economic Studies, v. 6o, n.1, p. 35-52, 1993. DOI: https://doi.org/10.2307/2297811

HANUSHEK, E.A., KIMKO, D.D. Schooling, Labor-Force Quality, and the Growth of Nations. The American Economic Review, v. 90, n. 5, p. 1184-1208, 2000. DOI: https://doi.org/10.1257/aer.90.5.1184

ISLAM, N. Growth Empirics: A Panel Data Approach. The Quarterly Journal of Economics, v. 110, n. 4, p. 1127-1170, 1995. DOI: https://doi.org/10.2307/2946651

HIRSCHMAN, Albert O. The strategy of economic development. New Haven: Yale University Press, 1958.

KRUGMAN, P. Geography and Trade. Published jointly by Leuven University press and the MIT Press, 1991. 
MANKIW, N.G.; ROMER, D. \& WEIL, D. A contribution to the empirics of economic growth. The Quarterly Journal of Economics, v. 107, n. 2, p. 407-437, 1992. DOI: https://doi.org/10.2307/2118477

MINCER, Jacob. Investment in human capital and personal income distribution. Journal of Political Economy, v. 66, n. 4, p. 22-50, 1958. DOI: https://doi.org/10.1086/258055

NAKABASHI, L.; FIGUEIREDO, L. Capital humano: uma nova proxy para incluir aspectos qualitativos. Cedeplar, Universidade Federal de Minas Gerais, 2005.

NAKABASHI, L.; SALVATO, M. A. Human capital quality in the Brazilian states. Revista Economia, v.8, n. 2, p. 211-229, 2007.

NELSON, R.; PHELPS, E. Investiment in Humans, Technological Diffusion and Economic Growth, American Economic Review, Paper and Proceedings, v.56, n.2, p. 69-75, 1966.

Organização para a Cooperação e Desenvolvimento Econômico - OCDE. The Well-Being of Nations: The Role of Human and Social Capital. Education and Skills. OCDE, Paris, 2001.

RESENDE, G., CARVALHO, T. S.; SAKOWSKI, P. Evaluating multiple spatial dimensions of economic growth in Brazil using spatial panel data models (1970-200o), Discussion Paper 1830, IPEA, 2014.

RAIHER, A.P. A evolução do capital humano e sua importância no crescimento econômico das microrregiões paranaenses no período de 1999 a 2006. Tese. Universidade Federal do Rio Grande do Sul. Faculdade de Ciências Econômicas. Programa de Pós-Graduação em Economia. Porto Alegre, 2009.

ROMER, P. Increasing Returns and Long-run Growth. Journal of Political Economy, v.94, p:1002-1037, 1986. DOI: https://doi.org/10.1086/261420

SCHULTZ, T. The Economic Value of Education, New York: Columbia University Press, 1963.

SOLOW, R.M. A Contribution to the Theory of Economic Growth. The Quarterly Journal of Economics, v. 70, n. 1, p. 65-94, 1956. DOI: https://doi.org/10.2307/1884513

WOESSMANN, L. (2003). Specifying human capital, Journal of Economic Surveys, v. 17, n.3, p. 239-270, 2003. DOI: https://doi.org/10.1111/1467-6419.00195 
Apêndice A

Critério de informação para a decisão do modelo espacial mais adequado

\begin{tabular}{ccccccccc}
\hline & \multicolumn{3}{c}{ Tabela 5- proxy QT } & \multicolumn{3}{c}{ Tabela 5 - proxy QL } \\
\hline & SAR & SEM & SEDM & $\begin{array}{c}\text { Durbin } \\
\text { erro }\end{array}$ & SAR & SEM & $\begin{array}{c}\text { durbin } \\
\text { Durbin } \\
\text { erro }\end{array}$ \\
\hline CritInf Akaike & -4464 & -4607 & -4763 & -4623 & -44441 & -4593 & -4784 & -4768 \\
Crit Inf Schwarz & -4424 & -4574 & -4697 & -4563 & -3301 & -4560 & -4717 & 4708 \\
\hline
\end{tabular}

Fonte: Geoda. 


\section{Apêndice B}

Teste de Heterocedasticidade, de normalidade dos resíduos e coeficiente I de Moran para o resíduo regressão MQO

\begin{tabular}{c|c|c|}
\hline & Proxy: escolaridade média & Proxy: [escolaridade média*(IDHConh+IDH long.)] \\
\hline I de Moran & $19^{*}$ & $18^{*}$ \\
Breuch-Pagan & $1979^{*}$ & $2704^{*}$ \\
Jarque-Bera & $30981^{*}$ & $68605^{*}$ \\
\hline
\end{tabular}

Fonte: GeodaSpace

Nota: nível de significância de $1 \%$ 
Apêndice C

Diagnóstico do modelo mais adequado - modelos Regimes espaciais

\begin{tabular}{ccc}
\hline & Proxy: escolaridade média & $\begin{array}{c}\text { Proxy: [escolaridade média*(IDHConh+IDH } \\
\text { long.)] }\end{array}$ \\
\hline${ }^{\mathrm{ML}} \rho^{\text {(defasagem) }}$ & $289^{*}$ & $294^{*}$ \\
$\mathrm{MLR} \rho^{\text {(defas. robusta) }}$ & $52^{*}$ & $57^{*}$ \\
$\mathrm{ML}^{\text {(erro) }}$ & $254^{*}$ & $259^{*}$ \\
${ }_{\mathrm{MLR}} \lambda^{\text {(erro robusto })}$ & $17^{*}$ & $22^{*}$ \\
\hline
\end{tabular}

Fonte: GeodaSpace

Nota: nível de significância de $1 \%$ 